\title{
Genomic structure and phylogenetic analysis of the luciferase gene of the firefly, Luciola lateralis (Coleoptera: Lampyridae)
}

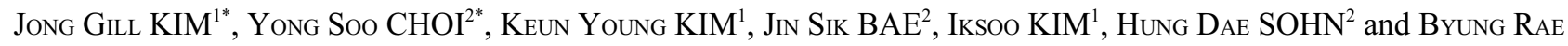 \\ $\mathrm{JIN}^{2 * *}$
}

${ }^{1}$ Department of Sericulture and Entomology, National Institute of Agricultural Science and Technology, Suwon 441-100, Korea

${ }^{2}$ College of Natural Resources and Life Science, Dong-A University, Busan 604-714, Korea

Key words. Firefly, Luciola lateralis, luciferase gene, genomic structure, phylogenetic analysis

\begin{abstract}
The complete nucleotide sequence and the exon-intron structure of the luciferase gene of the firefly, Luciola lateralis (Coleoptera: Lampyridae) is described. The luciferase gene of the L. lateralis firefly spans 1,971 bp and consists of six introns and seven exons coding for 548 amino acid residues. From samples collected at Boun and Muju, Korea, three isoforms, with identical exon-intron structure, named BU, MJ1 and MJ2, respectively, were obtained. Although the amino acid sequences of MJ1 and MJ2 were identical to those of known luciferase genes of Korean origin, the BU type was novel, differing from each of the MJ1 and MJ2 types by one amino acid. The luciferase sequences of the Korean samples, including those previously revealed, differed only by one - two amino acid residues, but differed by five - six amino acid residues from that of the luciferase gene recorded from specimens from Japan, which suggest genetic divergence has occurred in this species. Phylogenetic analyses using both amino acid and nucleotide sequences further showed that the luciferase gene of the Japanese L. lateralis firefly is genetically distinguishable from that of Korean L. lateralis, suggesting the presence of a genetic subdivision between the L. lateralis dwelling in the Korean Peninsula and Japanese Islands.
\end{abstract}

\section{INTRODUCTION}

Fireflies, luminescent insects, have species-specific flash patterns, which are used in sexual communication. In the light organ luciferase reacts with the substrate luciferin to produce bioluminescence. The firefly luciferase gene is widely used as a genetic marker or as a reporter gene in a variety of organisms including bacteria, plants and animals (De Wet et al., 1985, 1987; DiLella et al., 1988; Howard et al., 1988; Kondo et al., 1992; Miller et al., 1992; Jacobs et al., 1993; Bailey et al., 1994; Vikas et al., 1995; Lee et al., 2001). The enzymatic assay of luciferase is preferred because it is sensitive, rapid and a noninvasive method of quantification. More than 10 luciferase genes have been isolated from various firefly species. These include several species of the Luciolagroup such as L. lateralis (Tatsumi et al., 1992; Cho et al., 1999), L. cruciata (Masuda et al., 1989) and L. mingrelica (Devine et al., 1993) and the Hotaria-group such as H. parvula (Ohmiya et al., 1995), H. unmunsana (Choi et al., 2002), $H$. papariensis (Choi et al., 2003), and $H$. tsushimana (Choi et al., 2003). Ye et al. (1997) cloned and sequenced the luciferase from Photuris pennsylvanica of the Photurinae subfamily of the Lampyridae and compared it to that of several Luciolinae and one species of Hotaria. The ORF of the genes commonly encodes a protein of 548 amino acids and the percentage identity ranged from $80.8 \%$ to $99 \%$ among Luciolinae.

The subfamily Luciolinae, which is composed of seven genera including about 400 species, is divided into two tribes: Luciolini (Luciola, Pteroptyx, Pyrophanes, Colophotia, Lampyroides and Bourgeoisia) and Curtosini (Curtos). They are widely distributed in the world except for the polar ice caps and North and South of the Americas (McDermott, 1964). Luciola lateralis is common in Korea, Japan, and Siberia (Kim et al., 2001b; Suzuki, 2001). The luciferase gene of this species was sequenced independently by different laboratories using samples from different places (Tatsumi et al., 1992; Cho et al., 1999). Tatsumi et al. (1992) found one clone in samples collected in Japan. Cho et al. (1999) analyzed the genomic structure of the gene and found two structurally different genes: 128-bp deletion/insertion in the 5, upstream region, 16 nucleotides substitutions of $1,794 \mathrm{bp}$ in the 5' upstream, 7 nucleotides out of 1,644 bp in the coding region (resulted in two amino acid replacements), 4 out of 328 in the introns and 4 out of 419 in the noncoding 3' region.

In terms of phylogeny, several studies report genetic differentiation between geographic samples of $L$. lateralis. For example, Kim et al. (2000) sequenced a portion of mitochondrial (mt) 16S ribosomal RNA and found a substantial sequence divergence between Korean and GenBank-registered Japanese samples (AB009906; Suzuki, 1997). Suzuki et al. (2004) also found genetic divergence between Korean and Japanese samples using the mt cytochrome oxidase II (COII) gene. In addition, $\mathrm{Kim}$ et al. (2001a) reported that L. lateralis sampled in Korea has two distinct mtDNA clades. Although these

\footnotetext{
* These authors contributed equally to this paper.

** Corresponding author. E-mail: brjin@mail.donga.ac.kr
} 
TABLE 1. Origin of the L. lateralis fireflies with their amino acid sequence-based luciferase types, and GenBank accession numbers.

\begin{tabular}{|c|c|c|c|c|}
\hline Species & Origin & $\begin{array}{l}\text { Animal } \\
\text { number }\end{array}$ & $\begin{array}{l}\text { Luciferase } \\
\text { gene type }\end{array}$ & $\begin{array}{c}\text { GenBank } \\
\text { accession } \\
\text { number }\end{array}$ \\
\hline \multicolumn{5}{|c|}{ Sequences obtained in this study } \\
\hline \multirow[t]{4}{*}{ Luciola lateralis } & Boun, Korea & BU1 & $\mathrm{BU}$ & AY181996 \\
\hline & Boun, Korea & BU2 & $\mathrm{BU}$ & “ \\
\hline & Muju, Korea & MJ1 & MJ1 & AY192560 \\
\hline & Muju, Korea & MJ2 & MJ2 & AY192561 \\
\hline \multicolumn{5}{|c|}{ Sequences obtained from the GenBank } \\
\hline \multirow[t]{3}{*}{ Luciola lateralis } & Muju, Korea & - & MJ1 & $\begin{array}{l}\text { U51019, } \\
\text { U49181, } \\
\text { Z69619 }\end{array}$ \\
\hline & Muju, Korea & - & MJ2 & $\begin{array}{l}\text { U49182, } \\
\text { Z49891 }\end{array}$ \\
\hline & Japan & - & Japan & E05448 \\
\hline
\end{tabular}

studies attempted to clarify the phylogenetic relationships between Korean and Japanese populations (Kim et al., 2000; Suzuki, 2001; Suzuki et al., 2004) and within Korean populations (Kim et al., 2001a) only mt DNA genes were utilized as no data was available on the nuclear DNA then. If $L$. lateralis shows genetic differentiation geographically then the luciferase gene may be a useful portion of the nuclear DNA for drawing phylogenetic inferences about geographically separated populations. As fireflies use light signals in sexual communication and the luciferase gene is the sole enzyme interacting with the substrate luciferin to produce bioluminescence, the primary structure of the gene from local populations would be of interest for inferring phylogenetic relationships among geographically separated populations. This is because a local population, adapted to a given environment and with a specific flashing pattern may have a luciferase gene with a particular amino acid sequence.

In this study, we determined the genomic structure of the luciferase gene in L. lateralis from Boun and Muju, Korea. This was done because previous mitochondrial studies suggested the possibility of genetic divergence between the L. lateralis collected at these localities (Kim et al., 2001a). In addition, L. lateralis luciferase genes from Japanese (Tatsumi et al., 1992) and Muju populations (Cho et al., 1999) were included in the analysis for a better understanding of the phylogenetic relationships between the various luciferase genes of $L$. lateralis.

\section{MATERIAL AND METHODS}

\section{Animals \\ Information on the firefly, Luciola lateralis, used in this study is listed in Table 1. Two individuals of L. lateralis were col- lected both from Boun, Chungchungbook-do Province and Muju, Chollabuk-do Province, Korea, which are located in the mid-part of the Korean Peninsula.}

\section{Genomic DNA isolation and PCR of luciferase gene in the firefly}

Genomic DNA was extracted from the four L. lateralis using a Wizard ${ }^{\mathrm{TM}}$ Genomic DNA Purification Kit, according to the manufacturer's instructions (Promega, Madison, WI). The primers used for amplification of the genomic DNA encoding the luciferase were 5'GTGGAGTGTTTAGTTACAAGTGCGG-3' for the translational start sequence region and 5'-GAGTATAAACGATTTGAATCCCAC-3' for the 3' coding region, obtained from a previous study of a luciferase gene of $L$. lateralis (Tatsumi et al., 1992; Cho et al., 1999). After a 35cycle amplification $\left(94^{\circ} \mathrm{C}\right.$ for $30 \mathrm{~s} ; 48^{\circ} \mathrm{C}$ for $40 \mathrm{~s} ; 72^{\circ} \mathrm{C}$ for 2 min), PCR products were ethanol precipitated, centrifuged at $10,000 \times \mathrm{g}$ for $15 \mathrm{~min}$ and rinsed using $70 \%$ ethanol. These DNAs were analyzed with $1.0 \%$ agarose gel electrophoresis. The PCR products for sequencing were cloned in a pGem-T vector (Promega).

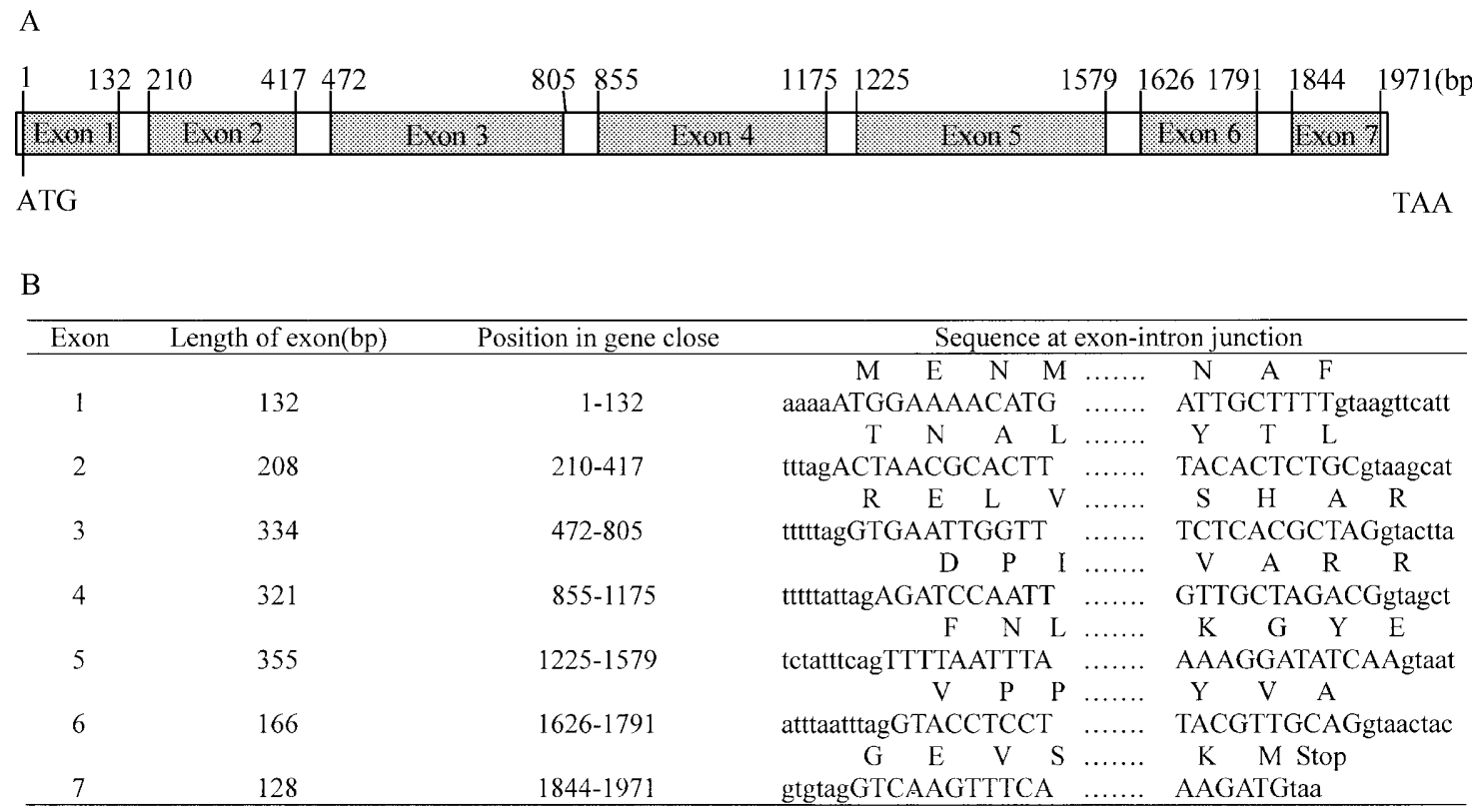

Fig 1. Genomic organization of the luciferase gene of the L. lateralis firefly. A - exon/intron structures. Numbers indicate the length (bp) of exons and introns; B - lengths of exons and exon/intron boundaries. 
TABLE 2. Pairwise comparisons between amino acid sequences of the L. lateralis luciferase genes obtained in this study and from the GenBank.

\begin{tabular}{lcccc}
\hline & 1 & 2 & 3 & 4 \\
\hline 1. L. lateralis BU (AY181996) & - & 0.002 & 0.002 & 0.011 \\
2. L. lateralis MJ1 (AY192560, U49181, U51019, Z69619) & 1 & - & 0.004 & 0.009 \\
3. L. lateralis MJ2 (AY192561, U49182, Z49891) & 1 & 2 & - & 0.009 \\
4. L. lateralis Japan (E05448) & 6 & 5 & 5 & - \\
\hline
\end{tabular}

Numbers above the diagonal are mean distance values; numbers below the diagonal are absolute distance values. The GenBank accession numbers within parenthesis indicate identical L. lateralis luciferase gene types, based on the amino-acid sequences: AY181996, AY192560 and AY192561 were obtained in this study.

\section{DNA sequencing and data analysis}

DNA sequencing was performed using an automatic sequencer (model 310 Genetic Analyzer; Perkin-Elmer Applied Biosystems, Foster City, CA). MacVector (ver. 6.5) was used to align the amino acid and nucleotide sequences of the luciferase gene. Maximum parsimony method was used to infer possible relationships among $L$. lateralis luciferase genes in terms of amino acid and nucleotide sequences, respectively, using PAUP (Phylogenetic Analysis using Parsimony) ver. 3.1 (Swofford, 1993). The accession numbers of the sequences in the GenBank are as follows: L. lateralis BU (this study), L. lateralis MJ1 (U51019, U49181, Z69619; Cho et al., 1999 and this study), L. lateralis MJ2 (U49182, Z49891; Cho et al., 1999 and this study), L. lateralis Japan (E05448; Tatsumi et al., 1992). Because the stop codon of L. lateralis Japan (E05448; Tatsumi et al., 1992) is not available in the GenBank, all the stop codons of the remaining luciferase genes were removed from the analyses. The luciferase gene of $L$. cruciata was utilized as an outgroup in both analyses (M26194; Masuda et al., 1989). The $L$. cruciata luciferase gene differed from L. lateralis luciferase sequences by $6.4 \%$ (35 amino acid residues) $-6.8 \%$ (37 amino acid residues) and 10\% (165 nucleotides) - 10.7\% (176 nucleotides). No weighting scheme was used for the nucleotide sequences. In both amino acid and nucleotide sequences, an heuristic search was performed and the reliability of the topology was tested by bootstrapping (1,000 iterations; Felsenstein, 1985).

\section{RESULTS}

\section{Genomic structure of the luciferase gene of the Luciola lateralis firefly}

The localities, from which the L. lateralis fireflies analyzed for the luciferase gene sequences come, are listed in Table 1. To identify the genomic structure of the luciferase gene of the L. lateralis firefly, a PCR primer set based on the sequences of the L. lateralis luciferase gene previously recorded by Tatsumi et al. (1992) and Cho et al. (1999) was designed. Using this primer set the luciferase gene of the genomic DNA of L. lateralis samples were successfully cloned and sequenced.

The luciferase genes of the four $L$. lateralis fireflies contain 1,971 bp, corresponding to 548 amino acids (Figs $1 \& 2$ ). Comparison of the genomic sequences with that of the previously published luciferase cDNA of the L. lateralis firefly indicates the presence of seven exons (Fig. 1A). From the known cDNA 5' site to the end of exon 7 the gene is $1,971 \mathrm{bp}$ long. Lengths of exons in luciferase genes were identical in all L. lateralis fireflies. The intron boundaries are listed in Fig. 1B. The consensus sequences, including an invariant GT at the intron 5, boundary and an invariant AG at its 3' boundary, were well conserved in all samples and those of the published L. lateralis luciferase gene.

\section{Isoforms}

From the samples a total of three types of luciferase gene, BU (from Boun, Korea), MJ1 (from Muju, Korea) and MJ2 (from Muju, Korea), were cloned (Table 1). The BU type luciferase gene of $L$. lateralis fireflies in the sample from Boun differed from the MJ1 and MJ2 types from Muju by one amino acid sequence, respectively. The amino acid sequences of the MJ1 type differed by two positions from the MJ2 type luciferase. The other GenBank-registered types (Cho et al., 1999; GenBank accession numbers Z69619, Z49891), which originated from Muju, Korea, are MJ1 and MJ2, respectively (Table 1). The luciferase gene of L. lateralis from Japan (Jpn type) differed by five or six amino acid positions from that from individuals from Muju and Boun in Korea. The

TABLE 3. Pairwise comparisons between nucleotide sequences of the L. lateralis luciferase genes obtained in this study and from the GenBank.

\begin{tabular}{lccccccc}
\hline & 1 & 2 & 3 & 4 & 5 & 6 & 7 \\
\hline 1. L. lateralis BU (AY181996) & - & 0.002 & 0.002 & 0.004 & 0.004 & 0.005 & 0.022 \\
2. L. lateralis MJ1 (AY192560) & 3 & - & 0.002 & 0.005 & 0.004 & 0.004 & 0.021 \\
3. L. lateralis MJ1 (U49181, U51019, Z69619) & 3 & 4 & - & 0.005 & 0.006 & 0.007 & 0.023 \\
4. L. lateralis MJ2 (AY192561) & 7 & 8 & 8 & - & 0.001 & 0.002 & 0.021 \\
5. L. lateralis MJ2 (U49182) & 7 & 6 & 10 & 2 & - & 0.001 & 0.021 \\
6. L. lateralis MJ2 (Z49891) & 8 & 7 & 11 & 3 & 1 & - & 0.020 \\
7. L. lateralis Japan (E05448) & 36 & 34 & 37 & 35 & 34 & 33 & - \\
\hline
\end{tabular}

Numbers above the diagonal are mean distance values; numbers below the diagonal are absolute distance values. The GenBank accession numbers within parenthesis indicate identical $L$. lateralis luciferase gene types, based on the amino-acid sequences: AY181996, AY192560 and AY192561 were obtained in this study. 


\begin{tabular}{|c|c|c|c|c|c|c|}
\hline \multirow[b]{2}{*}{ L. lateralis $\mathrm{BU}$} & & & & & & \multirow{2}{*}{$\begin{array}{r}60 \\
\text { VDYTYAEYLE }\end{array}$} \\
\hline & MENMDNDENI & VYGPKPFYPI & EEGSAGAQLR & KYMDRYAKLG & AIAFTNALTG & \\
\hline L. lateralis MJ1 & $\ldots \ldots \ldots$ & $\ldots \ldots \ldots$ & $\ldots \ldots \ldots$ & $\ldots \ldots \ldots$ & $\ldots \ldots \ldots$ & $\cdots$ \\
\hline L. lateralis MJ2 & $\ldots \ldots \ldots$ & $\ldots \ldots \ldots$ & $\ldots \ldots \ldots$ & $\ldots \ldots \ldots$ & $\ldots \ldots \ldots$ & $\ldots \ldots \ldots$ \\
\hline \multirow[t]{2}{*}{ L. lateralis Japan } & ... E... & ...Е... & $\ldots \ldots \ldots$ & $\ldots \ldots \ldots$ & $\ldots \ldots \ldots$ & $\cdots$ \\
\hline & & & 90 & & & 120 \\
\hline L. lateralis $\mathrm{BU}$ & KSCCLGEALK & NYGLVVDGRI & ALCSENCEEF & FIPVLAGLFI & GVGVAPTNEI & YTLRELVHSL \\
\hline L. lateralis MJ1 & $\ldots \ldots \ldots$ & $\ldots \ldots \ldots$ & $\ldots \ldots \ldots$ & $\ldots \ldots \ldots$ & $\ldots \ldots \ldots$ & $\ldots \ldots$ \\
\hline L. lateralis MJ2 & $\ldots \ldots \ldots$ & $\ldots \ldots \ldots$ & $\ldots \ldots \ldots$ & $\ldots \ldots \ldots$ & $\ldots \ldots \ldots$ & $\ldots \ldots$ \\
\hline \multirow[t]{2}{*}{ L. lateralis Japan } & $\ldots \ldots \ldots$ & $\ldots \ldots \ldots$ & $\ldots \ldots \ldots$ & $\ldots \ldots \ldots$ & $\ldots \ldots \ldots$ & ........ \\
\hline & & & 150 & & & \\
\hline L. lateralis $\mathrm{BU}$ & GISKPTIVFS & SKKGLDKVIT & VQKTVNAIKT & IVILDSKVDY & RGYQSMDNFI & KKNTPPGFKG \\
\hline L. lateralis $\mathrm{MJ} 1$ & $\ldots \ldots \ldots$ & $\ldots \ldots \ldots$ & $\ldots$. & $\ldots \ldots \ldots$ & $\ldots \ldots \ldots$ & $\ldots \ldots \ldots$ \\
\hline L. lateralis $\mathrm{MJ} 2$ & $\ldots \ldots \ldots$ & $\ldots \ldots \ldots$ & $\ldots \ldots \ldots$ & $\ldots \ldots \ldots$ & $\ldots \ldots \ldots$ & $\ldots \ldots$ \\
\hline \multirow[t]{2}{*}{ L. lateralis Japan } & $\ldots \ldots \ldots$ & $\ldots \ldots \ldots$ & $\ldots$.... & $\ldots \ldots \ldots$ & $\ldots \ldots \ldots$ & $\ldots . Q$. \\
\hline & & & 210 & & & 240 \\
\hline L. lateralis $\mathrm{BU}$ & SSFKTVEVNR & KEQVALIMNS & SGSTGLPKGV & QLTHENAVTR & FSHARDPIYG & NQVSPGTAIL \\
\hline L. lateralis $\mathrm{MJ} 1$ & $\ldots \ldots \ldots$ & $\ldots \ldots \ldots$ & $\ldots \ldots \ldots$ & $\ldots \ldots \ldots$ & $\ldots \ldots \ldots$ & $\ldots \ldots$ \\
\hline L. lateralis MJ2 & $\ldots \ldots \ldots$ & $\ldots \ldots \ldots$ & $\ldots \ldots \ldots$ & $\ldots \ldots \ldots$ & $\ldots \ldots \ldots$ & $\ldots \ldots$ \\
\hline \multirow[t]{2}{*}{ L. lateralis Japan } & $\ldots \ldots \ldots$ & $\cdots \cdots \cdots$ & $\cdots \cdots \cdots$ & $\cdots \cdots \cdots$ & $\ldots \ldots \ldots$ & $\ldots \ldots$ \\
\hline & & & 270 & & & 30 \\
\hline L. lateralis $\mathrm{BU}$ & TVVPFHHGFG & MFTTLGYLTC & GFRIVMLTKF & DEETFLKTLQ & DYKCSSVILV & PTLFAI LNRS \\
\hline L. lateralis MJ1 & $\ldots \ldots \ldots$ & $\ldots \ldots \ldots$ & $\ldots \ldots \ldots$ & $\ldots \ldots \ldots$ & $\ldots \ldots \ldots$ & $\ldots \ldots \ldots$ \\
\hline L. lateralis MJ2 & $\ldots \ldots \ldots$ & $\ldots \ldots \ldots$ & $\ldots \ldots \ldots$ & $\ldots \ldots \ldots$ & $\ldots \ldots \ldots$ & $\ldots \ldots$ \\
\hline \multirow[t]{2}{*}{ L. lateralis Japan } & $\ldots \ldots \ldots$ & $\ldots \ldots \ldots$ & $\ldots \ldots \ldots$ & $\ldots \ldots \ldots$ & $\ldots \ldots \ldots$ & $\ldots \ldots$ \\
\hline & & & 330 & & & 360 \\
\hline L. lateralis $\mathrm{BU}$ & ELLDKYDLSN & LVEIASGGAP & LSKEIGEAVA & RRFNLPGVRQ & GYGLTETTSA & IIITPEGDDK \\
\hline L. lateralis $\mathrm{MJ} 1$ & $\ldots \ldots \ldots$ & $\ldots \ldots \ldots$ & $\ldots \ldots \ldots$ & $\ldots \ldots \ldots$ & $\ldots \ldots \ldots$ & $\ldots \ldots$ \\
\hline L. lateralis MJ2 & $\ldots \ldots \ldots$ & $\ldots \ldots \ldots$ & $\ldots \ldots \ldots$ & $\ldots \ldots \ldots$ & $\ldots \ldots \ldots$ & $\ldots \ldots$ \\
\hline \multirow[t]{2}{*}{ L. lateralis Japan } & $\ldots \ldots \ldots$ & $\ldots \ldots \ldots$ & $\ldots \ldots \ldots$ & $\ldots \ldots \ldots$ & $\ldots \ldots \ldots$ & $\cdots \ldots \ldots$ \\
\hline & & & 390 & & & 420 \\
\hline L. lateralis $\mathrm{BU}$ & PGASGKVVPL & FKAKVIDLDT & KKTLGPNRRG & EVCVKGPMLM & KGYVDNPEAT & REIIDEEGWL \\
\hline L. lateralis $\mathrm{MJ} 1$ & $\ldots \ldots \ldots$ & $\ldots \ldots \ldots$ & $\ldots \ldots \ldots$ & $\ldots \ldots \ldots$ & $\ldots \ldots \ldots$ & $\ldots \ldots \ldots$ \\
\hline L. lateralis MJ2 & $\ldots \ldots \ldots$ & $\ldots \ldots \ldots$ & $\ldots \ldots \ldots$ & $\ldots \ldots \ldots$ & $\ldots \ldots \ldots$ & $\ldots \ldots \ldots$ \\
\hline \multirow[t]{2}{*}{ L. lateralis Japan } & $\ldots \ldots \ldots$ & $\ldots \ldots \ldots$ & $\ldots \ldots \ldots$ & $\cdots \cdots \cdots$ & $\ldots \ldots \ldots$ & $\ldots \ldots$ \\
\hline & & & 450 & & & 0 \\
\hline L. lateralis $\mathrm{BU}$ & HTGDIGYYDE & EKHFFIVDRL & KSLIKYKGYQ & VPPAELESVL & LQHPNIFDAG & VAGVPDPIAG \\
\hline L. lateralis $\mathrm{MJ} 1$ & $\ldots \ldots \ldots$ & $\ldots \ldots \ldots$ & $\ldots \ldots \ldots$ & $\ldots \ldots \ldots$ & $\ldots \ldots \ldots$ & $\ldots \ldots$ \\
\hline L. lateralis MJ2 & $\ldots \ldots \ldots$ & $\ldots \ldots \ldots$ & $\ldots \ldots \ldots$ & $\ldots \ldots \ldots$ & $\ldots \ldots \ldots$ & $\ldots \ldots$ \\
\hline \multirow[t]{2}{*}{ L. lateralis Japan } & $\cdots \ldots \ldots$ & $\cdots \cdots \cdots$ & $\ldots \ldots \ldots$ & $\ldots \ldots \ldots$ & $\ldots \ldots \ldots$ & $\ldots \ldots \ldots$ \\
\hline & & & 510 & & & \\
\hline L. lateralis $\mathrm{BU}$ & ELPGAVVVLE & KGKSMTEKKV & MDYVAGQVSN & AKRLRGGVRF & VDEVPKGLTG & KIDGKAIREI \\
\hline L. lateralis MJ1 & $\ldots \ldots \ldots$ & $\ldots \ldots \ldots$ & $\ldots \ldots \ldots$ & $\ldots \ldots \ldots$ & $\ldots \ldots \ldots$ & $\ldots \ldots \ldots$ \\
\hline L. lateralis $\mathrm{MJ} 2$ & $\ldots \ldots \ldots$ & . . WE. & $\ldots \ldots \ldots$ & $\ldots \ldots \ldots$ & $\ldots \ldots \ldots$ & $\ldots \ldots \ldots$ \\
\hline \multirow[t]{2}{*}{ L. lateralis Japan } & $\ldots \ldots \ldots$ & $\ldots \ldots E$ & $\ldots$..... & $\ldots \ldots \ldots$ & $\ldots \ldots \ldots$ & $\ldots \ldots$ \\
\hline & 548 & & & & & \\
\hline L. lateralis $\mathrm{BU}$ & LKKPVAKM & & & & & \\
\hline L. lateralis $\mathrm{MJ} 1$ & $\ldots \ldots$ & & & & & \\
\hline L. lateralis MJ2 & $\cdots$ & & & & & \\
\hline L. lateralis Japan & $\cdots$ & & & & & \\
\hline
\end{tabular}

Fig. 2. Alignment of amino acid sequences of the L. lateralis luciferase gene types obtained in this study and that of luciferase genes in the GenBank. The GenBank accession numbers of identical L. lateralis luciferase types are in parenthesis: L. lateralis BU type (AY181996 from this study), L. lateralis MJ1 type (AY192560 from this study; U49181, U51019, and Z69619 from Cho et al., 1999), L. lateralis MJ2 type (AY192561 from this study; U49182 and Z49891 from Cho et al., 1999), and L. lateralis Japan (E05448 from Tatsumi et al., 1992). Only amino acid positions that differ from the BU type are indicated.

amino acid sequence of the luciferase gene from BU type L. lateralis was $99.8 \%$ similar to that of $\mathrm{MJ} 1$ and $\mathrm{MJ} 2$ types, and $98.9 \%$ to the Jpn type (Table 2; Fig. 2).
The 1,644 bp of the nucleotide sequences corresponding to the seven exons of the L. lateralis luciferase genes are presented in Fig. 3. The two Boun samples had an identical type even at the nucleotide level (BU type).

Fig. 3. Alignment of seven nucleotide sequences of luciferase genes from $L$. lateralis fireflies. The four isoforms of the luciferase genes, defined by amino acid sequences (BU, MJ1, MJ2, and Japan types), turned out to be seven nucleotide sequence-based types. The GenBank numbers within parenthesis indicate identical sequences. Only nucleotide positions that differ from the BU type are indicated. 
L. lateralis BU (AY181996)

L. lateralis MJ1 (AY192560)

L. lateralis MJ2 (AY192560)

L. lateralis MJ2 (U49182)

L. lateralis MJ1 (U49181, U51019, Z69619 )

L. lateralis MJ2 (Z49891)

L. lateralis Japan (E05448)

L. lateralis BU (AY181996)

L. lateralis MJ1 (AY192560)

L. lateralis MJ2 (AY192560)

L. lateralis MJ2 (U49182)

L. lateralis MJ1 (U49181,

U51019, Z69619)

L. lateralis MJ2 (Z49891)

L. lateralis Japan (E05448)

L. lateralis BU (AY181996)

L. lateralis MJ1 (AY192560)

L. lateralis MJ2 (AY192560)

L. lateralis MJ2 (U49182)

L. lateralis MJ1 (U49181, U51019, Z69619)

L. lateralis MJ2 (Z49891)

L. lateralis Japan (E05448)

L. lateralis BU (AY181996)

L. lateralis MJ1 (AY192560)

L. lateralis MJ2 (AY192560)

L. lateralis MJ2 (U49182)

L. lateralis MJ1 (U49181,

U51019, Z69619)

L. lateralis MJ2 (Z49891)

L. lateralis Japan (E05448)

L. lateralis BU (AY181996)

L. lateralis MJ1 (AY192560)

L. lateralis MJ2 (AY192560)

L. lateralis MJ2 (U49182)

L. lateralis MJ1 (U49181,

U51019, Z69619)

L. lateralis MJ2 (Z49891)

L. lateralis Japan (E05448)

L. lateralis BU (AY181996)

L. lateralis MJ1 (AY192560)

L. lateralis MJ2 (AY192560)

L. lateralis MJ2 (U49182)

L. lateralis MJ1 (U49181, U51019, Z69619)

L. lateralis MJ2 (Z49891)

L. lateralis Japan (E05448)

L. lateralis BU (AY181996)

L. lateralis MJ1 (AY192560)

L. lateralis MJ2 (AY192560)

L. lateralis MJ2 (U49182)

L. lateralis MJ1 (U49181, U51019, Z69619)

L. lateralis MJ2 (Z49891)

L. lateralis Japan (E05448)

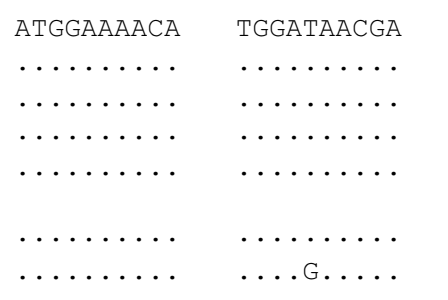

30

GAAGAGGGAT

$\ldots \ldots \ldots$

$\ldots \ldots \ldots$

$\ldots \ldots \ldots$

$\ldots \ldots \ldots$

$\ldots \ldots$

CTGCTGGAGC

$\ldots \ldots \ldots$

$\ldots \ldots \ldots$

$\ldots \ldots \ldots$

$\ldots \ldots \ldots$

$\ldots \ldots \ldots$

$\ldots \ldots .$.

GCAATTGCTT

TTACTAACGC

$\ldots \ldots \ldots$

$\ldots \ldots \ldots$

$\ldots \ldots \ldots$

$\ldots \ldots \ldots$

$\ldots \ldots \ldots$

$\ldots \ldots \ldots$

$\ldots \ldots \ldots$

. . .

$\ldots \ldots \ldots$

$\ldots \ldots \ldots$

AAATCATGCT

GTCTAGGAGA

\section{.......}

$\ldots \ldots$

$\ldots \ldots \ldots$

$\ldots \ldots \ldots$

.

$\ldots \ldots \ldots$

$\ldots \ldots \ldots$

$\ldots \ldots$

$\ldots \ldots \ldots$

$\ldots \ldots \ldots$

$\ldots \ldots \ldots$

GCGTTATGCA

$\ldots \ldots \ldots$

$\ldots \ldots \ldots$

$\ldots \ldots \ldots$

$\ldots \ldots \ldots$

$\ldots \ldots \ldots$

$\ldots \ldots$.

GGTGTCGGTG

$\ldots \ldots \ldots$

$\ldots \ldots \ldots$

$\ldots \ldots \ldots$

$\ldots \ldots \ldots$

$\ldots \ldots \ldots$

$\ldots \ldots$.

GGCATCTCTA

$\ldots \ldots \ldots$

. A.....

$\ldots \ldots . .$.

. A.....

$\ldots \ldots \ldots$

\section{0}

GTGAAAACTG

................

$\ldots \ldots \ldots$

$\ldots \ldots \ldots$

$\ldots \ldots \ldots$

$\ldots \ldots \ldots$

$\ldots \ldots \ldots$

TGGCTCCAAC

$\ldots \ldots \ldots$

$\ldots \ldots \ldots$

$\ldots \ldots \ldots$

$\ldots \ldots \ldots$

$\ldots \ldots \ldots$

$\ldots \ldots \ldots$

AGCCAACAAT

$\ldots \ldots \ldots$

$\ldots \ldots \ldots$

$\ldots \ldots \ldots$

$\ldots \ldots$.

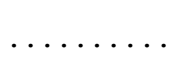

........

$\ldots \ldots$

$\ldots \ldots$

$\ldots \ldots \ldots$

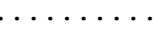

$\ldots \ldots \ldots$

330

TAATGAGATT

$\ldots \ldots \ldots$

$\ldots \ldots$

$\ldots \ldots \ldots$

$\ldots \ldots$

$\ldots \ldots \ldots$

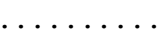

390

TGtATTtAGT

........

$\ldots \ldots \ldots$

$\ldots \ldots \ldots$

$\ldots \ldots \ldots$

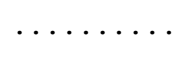

TaAAATATT

.......

$\ldots \ldots$

$\ldots \ldots \ldots$

$\ldots \ldots \ldots$

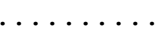

$\ldots \ldots \ldots$

90

ACAATTGCGC

$\ldots \ldots \ldots$

$\ldots \ldots \ldots$

$\ldots \ldots \ldots$

$\ldots \ldots$

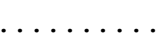

$\ldots \ldots$

GTATATGGTC

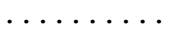

$\ldots \ldots \ldots$

$\ldots \ldots \ldots$

$\ldots \ldots \ldots$

$\ldots \ldots \ldots$

. G......

AAgTATATGG

$\ldots \ldots \ldots$

$\ldots \ldots \ldots$

$\ldots \ldots \ldots$

$\ldots \ldots \ldots$

$\ldots \ldots \ldots$

$\ldots \ldots$

150

ACTTACCGGT

$\ldots \ldots \ldots$

$\ldots \ldots \ldots$

$\ldots \ldots$

$\ldots \ldots \ldots$

$\ldots \ldots \ldots$

210

GGCTTTAAAG

$\ldots \ldots . . . .$.

$\ldots \ldots .$.

$\ldots \ldots$

$\ldots \ldots \ldots$

$\ldots \ldots \ldots$

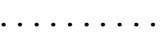

TGAAGAATTT

$\ldots \ldots \ldots$

GTCGATTATA

$\ldots . . . .$.

$\ldots \ldots \ldots$

$\ldots \ldots$.

$\ldots \ldots \ldots$

$\ldots \ldots \ldots$

AATtATGGTT

.........

$\ldots \ldots \ldots$

$\ldots \ldots \ldots$

$\ldots \ldots \ldots$

$\ldots \ldots \ldots$

$\ldots \ldots \ldots$

TTTATTCCTG

$\ldots \ldots \ldots$

$\ldots \ldots \ldots$

$\ldots \ldots \ldots$

TACACTCTGC

.........

$\ldots \ldots \ldots$

$\ldots \ldots \ldots$

$\ldots \ldots \ldots$

$\ldots \ldots \ldots$

$\ldots \ldots$....

TCTAAAAAAG

...........

60

СТАААССАТТ TTAССССАТT

$\ldots \ldots \ldots \ldots \ldots$

$\ldots \ldots \ldots \ldots$

$\ldots \ldots \ldots \ldots \ldots$

$\ldots \ldots \ldots \ldots$

$\ldots \ldots \ldots$

. G......

$\cdots$

120

ATCGATATGC AAAACTTGGC

$\ldots \ldots \ldots$

$\ldots \ldots \ldots \ldots \ldots$

$\ldots \ldots \ldots \ldots$

$\ldots \ldots \ldots \ldots$

$\ldots \ldots \ldots$

$\ldots \ldots \ldots$

$\cdots \cdots$

CGTACGCCGA

180

$\ldots \ldots \ldots$

$\ldots \ldots \ldots$

$\ldots \ldots \ldots$

$\ldots \ldots \ldots$

$\ldots \ldots \ldots$

$\ldots \ldots \ldots$

ATACTTAGAA

.........

$\ldots \ldots \ldots$

$\ldots \ldots \ldots$

$\ldots \ldots \ldots$

$\ldots \ldots \ldots$

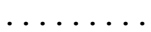

TGGTTGTTGA

240

.........

$\ldots \ldots \ldots$

$\ldots \ldots \ldots$

$\ldots \ldots \ldots$

$\ldots \ldots \ldots$

$\ldots \ldots \ldots$

TATTAGCCGG

.........

.........

$\ldots \ldots \ldots$

$\ldots \ldots \ldots$

$\ldots \ldots$

GTGAATTGGT

........

$\ldots \ldots \ldots$

$\ldots \ldots \ldots$

$\ldots \ldots \ldots$

$\ldots \ldots \ldots$

GATTAgATAA

.......... 


\author{
L. lateralis BU (AY181996) \\ L. lateralis MJ1 (AY192560) \\ L. lateralis MJ2 (AY192560) \\ L. lateralis MJ2 (U49182) \\ L. lateralis MJ1 (U49181, \\ U51019, Z69619) \\ L. lateralis MJ2 (Z49891) \\ L. lateralis Japan (E05448)
}

L. lateralis BU (AY181996)

L. lateralis MJ1 (AY192560)

L. lateralis MJ2 (AY192560)

L. lateralis MJ2 (U49182)

L. lateralis MJ1 (U49181, U51019, Z69619)

L. lateralis MJ2 (Z49891)

L. lateralis Japan (E05448)

L. lateralis BU (AY181996)

L. lateralis MJ1 (AY192560)

L. lateralis MJ2 (AY192560)

L. lateralis MJ2 (U49182)

L. lateralis MJ1 (U49181, U51019, Z69619)

L. lateralis MJ2 (Z49891)

L. lateralis Japan (E05448)

L. lateralis BU (AY181996)

L. lateralis MJ1 (AY192560)

L. lateralis MJ2 (AY192560)

L. lateralis MJ2 (U49182)

L. lateralis MJ1 (U49181, U51019, Z69619)

L. lateralis MJ2 (Z49891)

L. lateralis Japan (E05448)

L. lateralis BU (AY181996)

L. lateralis MJ1 (AY192560)

L. lateralis MJ2 (AY192560)

L. lateralis MJ2 (U49182)

L. lateralis MJ1 (U49181, U51019, Z69619)

L. lateralis MJ2 (Z49891)

L. lateralis Japan (E05448)

L. lateralis BU (AY181996)

L. lateralis MJ1 (AY192560)

L. lateralis MJ2 (AY192560)

L. lateralis MJ2 (U49182)

L. lateralis MJ1 (U49181, U51019, Z69619)

L. lateralis MJ2 (Z49891)

L. lateralis Japan (E05448)

L. lateralis BU (AY181996)

L. lateralis MJ1 (AY192560)

L. lateralis MJ2 (AY192560)

L. lateralis MJ2 (U49182)

L. lateralis MJ1 (U49181,

U51019, Z69619)

L. lateralis MJ2 (Z49891)

L. lateralis Japan (E05448)
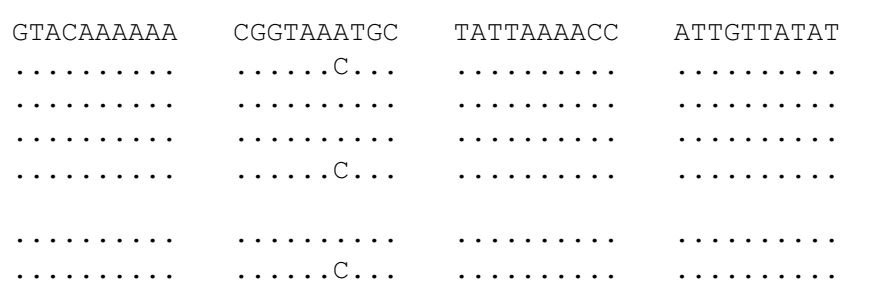

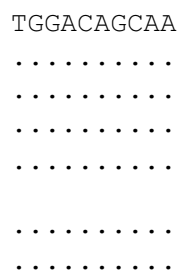

510

AGAGGATATC

AATCAATGGA

...G....

........

........

$\ldots \ldots \ldots$

$\ldots \ldots \ldots$

$\ldots \ldots \ldots$

.... т...

.........

...G....

........

...G....

... с....

TCAAGTTTTA

AAACTGTAGA

........

$\ldots \ldots$

$\ldots \ldots$

.........

..........

$\ldots \ldots \ldots$

$\ldots \ldots \ldots$

.......

TCGGGTTCCA

$\ldots \ldots \ldots$

CCGGTTTGCC

.........

$\ldots \ldots$

$\ldots \ldots \ldots$

$\ldots \ldots \ldots$

$\ldots \ldots \ldots$

.......

\section{.........}

$\ldots \ldots \ldots$

..........

$\ldots \ldots \ldots$

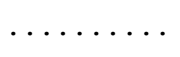

$\cdots \ldots \ldots$

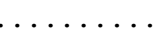

TTTTCTCACG

CTAGAGATCC

........

.........

$\ldots \ldots \ldots$

$\ldots \ldots$

........

$\ldots \ldots \ldots$

$\ldots \ldots \ldots$

$\ldots \ldots \ldots$

$\ldots \ldots \ldots$

$\ldots \ldots \ldots$

..........

ACTGTAGTAC

CATTCCATCA

.........

.........

.........

$\ldots \ldots \ldots$

$\ldots \ldots \ldots$

$\ldots \ldots \ldots$

$\ldots \ldots \ldots$

.........

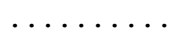

$\ldots \ldots \ldots$

.........

GGTTTTCGTA TTGTCATGTT AACAAAATTT

.........

$\ldots \ldots \ldots$

$\ldots \ldots \ldots$

$\ldots \ldots \ldots$

$\ldots \ldots \ldots$

........
.........

$\cdots \ldots \ldots$

....

$\ldots \ldots \ldots$

$\ldots \ldots \ldots$

$\ldots \ldots$
CAACTTTATT

$\ldots \ldots \ldots$

n......

$\ldots \ldots \ldots$

$\ldots \ldots \ldots$

$\ldots \ldots \ldots$

$\ldots \ldots \ldots$

570

AGTTAACCGC

$\ldots \ldots .$.

........

........

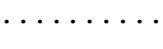

........

$\ldots \ldots \ldots$

AAAAGGTGTG

.........

.........
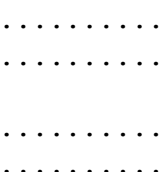

690

AATTTATGGA

.........

$\ldots \ldots$

$\ldots \ldots$
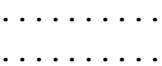

750

TGGTTTTGGT

.........

..........

$\ldots \ldots \ldots$
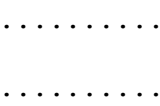

.

810

AAAAAAAACA

.........

$\ldots \ldots \ldots$

.........

........

$\ldots \ldots \ldots$

.

AAAGAACAAG

.........

$\ldots \ldots \ldots$

$\ldots \ldots$.

$\ldots \ldots \ldots$

........

$\ldots \ldots \ldots$

CAACTTACTC

.........

.........

$\ldots \ldots \ldots$

.........

$\ldots \ldots$.

AATCAAGTTT

.......

.........

$\ldots \ldots \ldots$

.........

.........

.c......

ATGTTTACTA

.........

$\ldots \ldots \ldots$

$\ldots \ldots \ldots$

$\ldots \ldots \ldots$

$\ldots \ldots \ldots$

........

.........

.........

$\ldots \ldots \ldots$

...G.....

GACGAAGAAA

.........

..........

$\ldots$

$\ldots \ldots \ldots$

........

......G.
.........
CTCCACCAGG

.........

.........

$\ldots \ldots \ldots$

$\ldots \ldots \ldots$

.........

.......

$\cdots \cdots$

TTGCTCTCAT

........

$\ldots \ldots \ldots$

.......

$\ldots \ldots \ldots$

........

...... T.

ACGAAAATGC

........

$\ldots \ldots \ldots$

$\ldots \ldots \ldots$

.........

. T......

$\ldots \ldots$

CACCAGGCAC

........

$\ldots \ldots \ldots$

$\ldots \ldots \ldots$

$\ldots \ldots \ldots$

.........

$\ldots \ldots \ldots$

CTTTAGGCTA

.........

$\ldots \ldots \ldots$

$\ldots \ldots$

$\ldots \ldots \ldots$

$\ldots \ldots \ldots$

........

........

CGTTTTTAAA AACACTGCAA

$\ldots \ldots \ldots \ldots \ldots$

$\ldots \ldots \ldots \ldots$

$\ldots \ldots \ldots$

$\ldots \ldots \ldots$

. T......

- w. n

900 


\begin{abstract}
L. lateralis BU (AY181996)
L. lateralis MJ1 (AY192560)

L. lateralis MJ2 (AY192560)

L. lateralis MJ2 (U49182)

L. lateralis MJ1 (U49181, U51019, Z69619)

L. lateralis MJ2 (Z49891)

L. lateralis Japan (E05448)
\end{abstract}

L. lateralis BU (AY181996)

L. lateralis MJ1 (AY192560)

L. lateralis MJ2 (AY192560)

L. lateralis MJ2 (U49182)

L. lateralis MJ1 (U49181, U51019, Z69619)

L. lateralis MJ2 (Z49891)

L. lateralis Japan (E05448)

L. lateralis BU (AY181996)

L. lateralis MJ1 (AY192560)

L. lateralis MJ2 (AY192560)

L. lateralis MJ2 (U49182)

L. lateralis MJ1 (U49181, U51019, Z69619)

L. lateralis MJ2 (Z49891)

L. lateralis Japan (E05448)

L. lateralis BU (AY181996)

L. lateralis MJ1 (AY192560)

L. lateralis MJ2 (AY192560)

L. lateralis MJ2 (U49182)

L. lateralis MJ1 (U49181,

U51019, Z69619)

L. lateralis MJ2 (Z49891)

L. lateralis Japan (E05448)

L. lateralis BU (AY181996)

L. lateralis MJ1 (AY192560)

L. lateralis MJ2 (AY192560)

L. lateralis MJ2 (U49182)

L. lateralis MJ1 (U49181, U51019, Z69619)

L. lateralis MJ2 (Z49891)

L. lateralis Japan (E05448)

L. lateralis BU (AY181996)

L. lateralis MJ1 (AY192560)

L. lateralis MJ2 (AY192560)

L. lateralis MJ2 (U49182)

L. lateralis MJ1 (U49181, U51019, Z69619)

L. lateralis MJ2 (Z49891)

L. lateralis Japan (E05448)

L. lateralis BU (AY181996)

L. lateralis MJ1 (AY192560)

L. lateralis MJ2 (AY192560)

L. lateralis MJ2 (U49182)

L. lateralis MJ1 (U49181,

U51019, Z69619)

L. lateralis MJ2 (Z49891)

L. lateralis Japan (E05448)

\section{GATTACAAAT \\ ......... \\ $\ldots \ldots \ldots$ \\ $\ldots \ldots \ldots$ \\ ........ \\ $\ldots \ldots \ldots$ \\ $\ldots \ldots \ldots$ \\ GAATTACTCG \\ ........ \\ $\ldots \ldots \ldots$ \\ $\ldots \ldots \ldots$ \\ $\ldots \ldots \ldots$

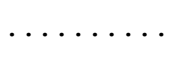 \\ $\ldots \ldots \ldots$ \\ TTATCAAAAG \\ ........ \\ $\ldots \ldots \ldots$ \\ $\ldots \ldots \ldots$ \\ ........ \\ $\ldots \ldots \ldots$ \\ .... т... \\ GTTCAAGTGT \\ ......... \\ ...т.... \\ ...т.... \\ .......... \\ ... т.... \\ ....... . \\ ATAAATATGA \\ ......... \\ $\ldots \ldots \ldots$ \\ $\ldots \ldots \ldots$ \\ $\ldots \ldots \ldots$ \\ $\ldots \ldots \ldots$ \\ $\ldots \ldots \ldots$ \\ AAATTGGTGA \\ ......... \\ $\ldots \ldots$ \\ $\ldots \ldots$. \\ ......... \\ $\ldots \ldots \ldots$ \\ $\ldots \ldots \ldots$}

GGCTATGGTT

........

.т.....

. т.....

$\ldots \ldots \ldots$

.т.....

........

CCAGGTGCTT

........

.........

..........

$\ldots \ldots$.

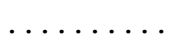

.

AAAAAAACTT

.........

.........

..........

$\ldots \ldots \ldots$

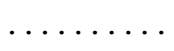

$\ldots \ldots \ldots$

AAAgGTTATG

.........

$\ldots \ldots \ldots$

.........

$\ldots \ldots \ldots$

$\ldots \ldots \ldots$

.......
TAACAGAAAC

..........

$\ldots \ldots$

$\ldots \ldots \ldots$

$\ldots \ldots \ldots$

$\ldots \ldots$

CTGGCAAAGT

.........

.........

$\ldots \ldots$

........

$\ldots \ldots \ldots$

.........

TGGGCCCGAA

.........

..........

$\ldots \ldots \ldots$

........

$\ldots \ldots \ldots$

.........

TAGATAATCC

.........

$\ldots \ldots \ldots$

..........

....... $\ldots \ldots \ldots$
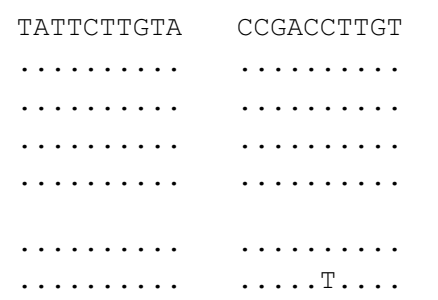

TTGCAATTCT

........

$\ldots \ldots \ldots$

.........

.........

$\ldots \ldots \ldots$

.........

930

TTTATCAAAT

.........

$\ldots \ldots \ldots$

$\ldots \ldots \ldots$

$\ldots \ldots$.
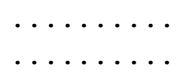

TTAGTTGAAA

TTGCATCTGG

........

$\ldots \ldots \ldots$

$\ldots \ldots \ldots$

.........

$\ldots \ldots \ldots$

990

AGCTGTTGCT

........

.........

$\ldots \ldots \ldots$

........

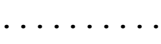

$\ldots \ldots \ldots$

AGACGTTTTA

.........

.........

.........

$\ldots \ldots \ldots$

$\ldots \ldots \ldots$

$\ldots \ldots \ldots$

1050

AACCTCTGCA

.........

..........

$\ldots \ldots \ldots$

.........

$\ldots \ldots$

$\ldots \ldots \ldots$

1110

TGTGCCATTA

.........

$\ldots \ldots \ldots$

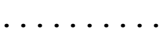

$\ldots \ldots \ldots$

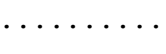

1170

CAGACGTGGA

........

$\ldots \ldots \ldots$

$\ldots \ldots \ldots$
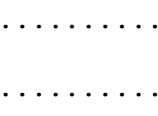

1230

AGAAGCAACA

.......

..........

.........

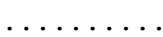

$\ldots \ldots \ldots$

........

1290
.........

$\ldots \ldots \ldots$

$\ldots \ldots \ldots$

$\ldots \ldots \ldots$

.........

.....

TTTAAAGCAA

.........

$\ldots \ldots \ldots$

$\ldots \ldots \ldots$

.........

$\ldots \ldots \ldots$

.

GAAGTTTGTG

.........

.........

........

.........

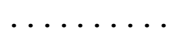

. . . . . . .

AgAgAAATtA

........

.........

$\ldots \ldots \ldots$

.........

$\ldots \ldots \ldots$

......
ATTATTATTA

........

$\cdots \cdots$

........

$\ldots \ldots \ldots$

$\ldots \ldots \ldots$

.........

..........

$\ldots \ldots \ldots$

$\ldots \ldots \ldots$

$\ldots \ldots \ldots$

$\ldots \ldots$

CACCAGAAGG

TAATAGAAGT

.........

$\ldots \ldots \ldots$

..........

.........

.........

$\ldots \ldots \ldots$

960

CGGAGCACCT

$\ldots \ldots \ldots$

1020

ATTTACCGG TGTTCGTCAA

........

$\ldots \ldots \ldots$

$\ldots \ldots \ldots$

$\ldots \ldots \ldots$

$\ldots \ldots \ldots$

$\ldots \ldots \ldots$

1080 


\author{
L. lateralis BU (AY181996) \\ L. lateralis MJ1 (AY192560) \\ L. lateralis MJ2 (AY192560) \\ L. lateralis MJ2 (U49182) \\ L. lateralis MJ1 (U49181, \\ U51019, Z49891) \\ L. lateralis MJ2 (Z69619) \\ L. lateralis Japan (E05448)
}

L. lateralis BU (AY181996)

L. lateralis MJ1 (AY192560)

L. lateralis MJ2 (AY192560)

L. lateralis MJ2 (U49182)

L. lateralis MJ1 (U49181,

U51019, Z69619)

L. lateralis MJ2 (Z49891)

L. lateralis Japan (E05448)

L. lateralis BU (AY181996)

L. lateralis MJ1 (AY192560)

L. lateralis MJ2 (AY192560)

L. lateralis MJ2 (U49182)

L. lateralis MJ1 (U49181, U51019, Z69619)

L. lateralis MJ2 (Z49891)

L. lateralis Japan (E05448)

L. lateralis BU (AY181996)

L. lateralis MJ1 (AY192560)

L. lateralis MJ2 (AY192560)

L. lateralis MJ2 (U49182)

L. lateralis MJ1 (U49181, U51019, Z69619)

L. lateralis MJ2 (Z49891)

L. lateralis Japan (E05448)

L. lateralis BU (AY181996)

L. lateralis MJ1 (AY192560)

L. lateralis MJ2 (AY192560)

L. lateralis MJ2 (U49182)

L. lateralis MJ1 (U49181, U51019, Z69619)

L. lateralis MJ2 (Z49891)

L. lateralis Japan (E05448)

L. lateralis BU (AY181996)

L. lateralis MJ1 (AY192560)

L. lateralis MJ2 (AY192560)

L. lateralis MJ2 (U49182)

L. lateralis MJ1 (U49181, U51019, Z69619)

L. lateralis MJ2 (Z49891)

L. lateralis Japan (E05448)

L. lateralis BU (AY181996)

L. lateralis MJ1 (AY192560)

L. lateralis MJ2 (AY192560)

L. lateralis MJ2 (U49182)

L. lateralis MJ1 (U49181,

U51019, Z69619)

L. lateralis MJ2 (Z49891)

L. lateralis Japan (E05448)
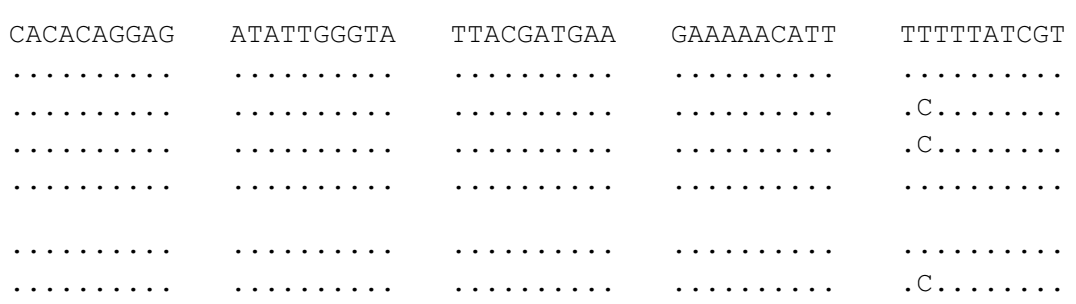

GGATCGTTTG

........

$\ldots \ldots \ldots$

$\ldots \ldots \ldots$

$\ldots \ldots \ldots$

$\ldots \ldots \ldots$

1350

AAGTCTTTAA

TCAAATACAA

AGGATATCAA

.........

$\ldots \ldots \ldots$

$\ldots \ldots \ldots$

........

.........

.........

$\ldots \ldots \ldots$

$\ldots \ldots \ldots$

........

$\ldots \ldots \ldots$

$\ldots \ldots \ldots$

$\ldots \ldots \ldots$

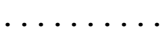

........ $\ldots \ldots \ldots$

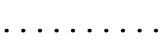

TTGCAACATC

CAAATATTTT

1410

TGATGCCGGC

........

........

$\ldots \ldots \ldots$

$\ldots \ldots \ldots$

.......

$\ldots \ldots \ldots$

$\ldots \ldots \ldots$

$\ldots \ldots \ldots$

$\ldots \ldots \ldots$

........

.........

(n)

$\ldots \ldots$

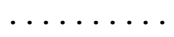

$\ldots \ldots \ldots$

GAGCTTCCGG

GAGCTGTTGT

1470

TGTACTTGAA

........

........

$\ldots \ldots \ldots$

$\ldots . . . .$.

$\ldots \ldots \ldots$

.........

$\ldots \ldots \ldots$

.........

$\ldots \ldots \ldots$

$\ldots \ldots \ldots$

$\ldots \ldots \ldots$

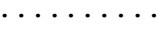

...............

ATGGATtACG

........

$\ldots \ldots \ldots$

$\ldots \ldots \ldots$

$\ldots \ldots \ldots$

........

GTGGACGAAG

........

$\ldots \ldots \ldots$

$\ldots \ldots \ldots$

$\ldots \ldots \ldots$

.......

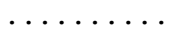

$\ldots \ldots \ldots$

CTAAAGAAAC

........

$\ldots \ldots \ldots$

$\ldots \ldots \ldots$

.........

.........

. G......
.........

TTGCAGGTCA

........

$\ldots \ldots \ldots$

$\ldots \ldots \ldots$

.............

$\ldots \ldots \ldots$

...TA...

TACCTAAAGG

.........

$\ldots \ldots \ldots$

$\ldots \ldots \ldots$

$\ldots \ldots \ldots$

........

CAGTTGCTAA

.........

$\ldots \ldots \ldots$

........

........

1530

AGTTTCAAAT

.........

$\ldots \ldots \ldots$

.........

$\ldots \ldots \ldots$

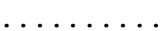

1590

TCTTACTGGT

........

$\ldots \ldots \ldots$

$\ldots \ldots \ldots$

$\ldots \ldots \ldots$

.........

... с.....

1644

GATG

....

$\ldots$

$\ldots$

$\ldots$

$\ldots \ldots \ldots \ldots$

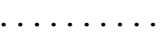

GTACCTCCTG

.........

........

........

........

........

$\ldots$.....

GTTGCTGGCG

........

..........

$\ldots \ldots \ldots$

........

.........

.........

1380

CTGAATtAgA AtCTGTTCtT

\section{............}

$\ldots \ldots \ldots \ldots$

$\ldots \ldots \ldots \ldots \ldots$

$\ldots \ldots \ldots$

$\ldots \ldots \ldots$

$\ldots \ldots \ldots$

$\ldots \ldots \ldots$

$\ldots \ldots \ldots$

1440

TTCCAGATCC

........

$\ldots \ldots \ldots$

$\ldots \ldots \ldots$

$\ldots \ldots \ldots$

$\ldots \ldots \ldots$

$\ldots \ldots \ldots$

TATAGCTGGT

........

$\ldots \ldots \ldots$

$\ldots \ldots \ldots$

$\ldots \ldots \ldots$

$\ldots \ldots \ldots$

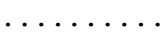

AAAGGAAAAT

CTATGACTGA

........

$\ldots \ldots \ldots$

.

$\ldots \ldots \ldots$

$\ldots \ldots \ldots$

- . n .

$\ldots \ldots \ldots$

$\ldots \ldots \ldots$

.

.

$\cdots$

GCAAAACGTT

TGCGTGGTGG

........

.........

$\ldots \ldots \ldots$

$\ldots \ldots \ldots$

$\ldots \ldots \ldots$

$\ldots \ldots \ldots$

.........

........

.........

AAAATTGATG

GTAAAGCAAT

..........

........

.........

..........

$\ldots \ldots \ldots$

.........

$\ldots \ldots \ldots$

$\ldots \ldots \ldots$

........
1620

1500

AAAAAAAGTA

........

...G.....

.........

....G....

1560

TGTCCGTTTT

.

$\ldots \ldots \ldots$

$\ldots \ldots \ldots$

TAGAGAAATA

........

$\ldots \ldots \ldots$

.........

$\ldots \ldots \ldots$

$\ldots \ldots \ldots$
....G.... 


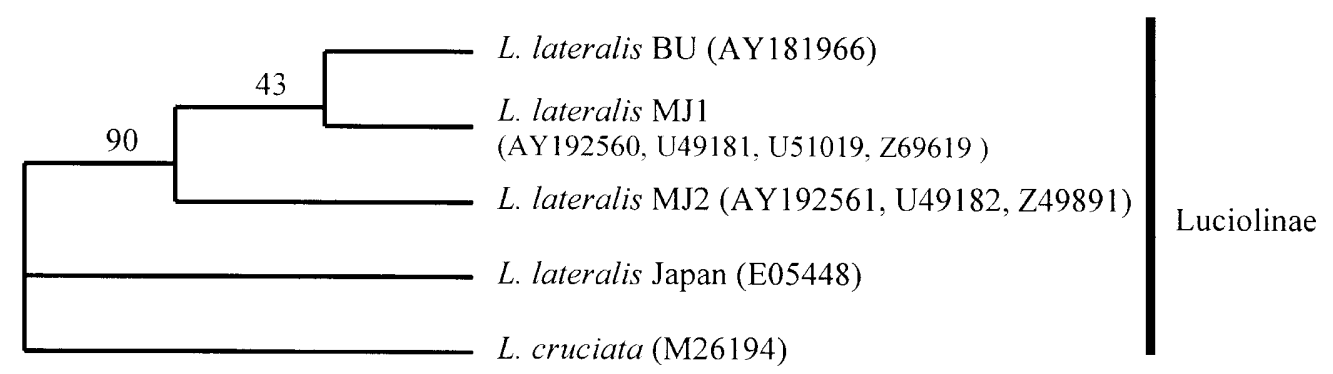

Fig. 4. A phylogenetic tree of aligned amino acid sequences of the L. lateralis firefly luciferase. The tree was obtained by bootstrap analysis with the option of heuristic search and the numbers on the branches represent bootstrap values for 1,000 replicates. The outgroup chosen was Luciola cruciata (Masuda et al., 1989). The GenBank numbers within parenthesis indicate identical sequences.

However, the two amino acid-based Muju types (MJ1 and MJ2), recorded in this study and that of Cho et al. (1999) differed at the nucleotide level. Thus, these appear to be at least seven nucleotide sequence-based luciferase gene types, including one Japanese type (Table 3; Fig. 3). The sequence divergence among the six Korean nucleotide types ranged from $0.1 \%$ (one nucleotide) to $0.7 \%$ (11 nucleotides). However, when the Japanese type was included, the sequence divergence ranged from 33 to 37 nucleotides $(2.0 \%-2.3 \%)$ and the Japanese type was more distant from the Korean types than they were from one another (Table 3).

\section{Phylogenetic analysis}

To illustrate the phylogenetic relationships among $L$. lateralis luciferase genes of different geographical origin, a maximum parsimony analysis was performed on the amino acid and nucleotide sequences using PAUP software (Figs $4 \& 5$ ). The analysis using amino acid sequences revealed that the Korean types (BU, MJ1, and MJ2 types) formed a strong subgroup ( $90 \%$ of bootstrap value), with a close relationship between the BU and MJ1 types, and excluded the Japanese type from the Korean types (Fig. 4). This suggests that the Japanese and Korean L. lateralis have diverged genetically. In terms of nucleotide sequences the two Muju types defined by amino acid sequences (MJ1 and MJ2 types) were subdivided into two groups and the Boun type grouped together with the MJ1 type. There are also two groups in the GenBankregistered sequences from Muju, Korea, which also suggests the presence there of phylogenetically divergent groups. As shown by the analysis using amino acid sequences, the Japanese luciferase gene is not grouped with the Korean types (Fig. 4), suggesting a genetic divergence between the populations in Korea and Japan. As an alternative to the parsimony analysis, FITSCH for amino acid and NEIGHBOR for nucleotide sequences incorporated in PHYLIP (Phylogeny Inference Package) ver. 3.5c (Felsenstein, 1993) were also utilized, but overall the topology was identical with that obtained using the maximum parsimony method (data not shown).

\section{DISCUSSION}

It is well known that the firefly uses luciferase to produce bioluminescence. The firefly luciferase genes have been isolated from various firefly species and are widely used as a genetic marker or reporter gene in a variety of systems. In this study, we cloned the luciferase genomic DNAs from four individuals of the firefly L. lateralis. The length of the gene is identical in four individuals from two populations: it contains $1,644 \mathrm{bp}$, corresponding to 548 amino acids. The previous studies on luciferase genes of $L$. lateralis also indicate they are all the same length (Tatsumi et al., 1992; Cho et al., 1999). This feature is conserved among species of the Luciolinae subfamily (Masuda et al., 1989; Devine et al., 1993; Choi et al., 2002, 2003). The analysis of the exon/intron boundary suggested the presence of seven exons in the luciferase gene of $L$. lateralis. The total length of the luciferase gene, including exons and introns, is 1,971-bp, and the exon/intron boundary is identical in all samples. However, there are isoforms in the four individuals of $L$. lateralis collected from the two Korean localities: $\mathrm{BU}$ type from Boun and MJ1 and MJ2 types from Muju. The luciferase gene in several firefly species is known to be polymorphic (Wood et al., 1989; Tatsumi et al., 1992; Zenno et al., 1994; Ye et al., 1997; Cho et al., 1999). The MJ1 and MJ2 types are identical in terms of the amino acid sequences of the genes with those previously collected from Muju, Korea (Cho et al., 1999): MJ1 to Luc2 and MJ1 to Luc2. However, the luciferase gene of BU type is novel. In terms of nucleotide sequences, the amino acid-based MJ1 and MJ2 obtained in this study are different from the MJ1 and MJ2 recorded by Cho et al. (1999). Furthermore, these Korean isoforms are not identical to the Japanese isoform (Tatsumi et al., 1992). So far, the L. lateralis firefly is known to have four different amino acid-based luciferase genes: three isoforms from Korean localities and one from a Japanese locality.

The deduced amino acid sequences of the luciferase gene of the Korean $L$. lateralis firefly are very similar. The amino acid sequence divergence ranged from $0.2 \%$ to $0.4 \%$ (one - two amino acid residues). When the L. lateralis collected in Japan (Tatsumi et al., 1992) is included, the divergence ranged from $0.9 \%$ to $1.1 \%$ (five - six amino acid residues) and these values are obtained only when Japanese and Korean isoforms are compared. This suggests a genetic discontinuity between the Korean and Japanese L. lateralis populations. 


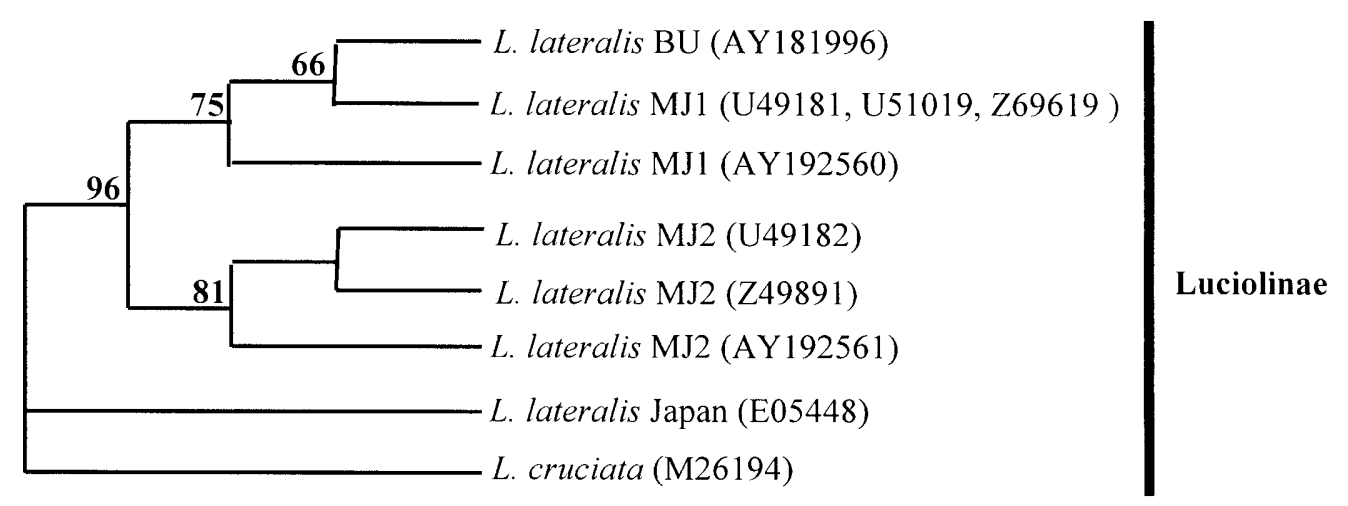

Fig. 5. A phylogenetic tree of aligned nucleotide sequences of the L. lateralis firefly luciferase. The tree was obtained by bootstrap analysis with the option of heuristic search and the numbers on the branches represent bootstrap values for 1,000 replicates. The outgroup chosen was Luciola cruciata (Masuda et al., 1989). The GenBank numbers within parenthesis indicate identical sequences. Because the stop codon of L. lateralis Japan (E05448; Tatsumi et al., 1992) is not available in the GenBank, all the stop codons of the remaining luciferase genes were removed from this analysis.

A phylogenetic analysis based on the luciferase genes of L. lateralis was performed in order to determine the genetic relationships among the genes. In the maximum parsimony analysis, luciferase gene isoforms in the samples from Korea formed a monophyletic group $(90 \%$ bootstrap value) distinct from those from Japan (Fig. 4).

As the luciferase gene is involved in sexual communication differences or similarities in its structure could determine the boundary between species (Ohmiya et al., 1995; Lee et al., 2001; Choi et al., 2002, 2003). The similarity of the amino acid sequences of the luciferases of the L. lateralis from Korea (Table 2; one - two amino acid residue difference) and that they differed from that of the L. lateralis luciferase from Japan (Fig. 3) suggest that there is a distinct genetic boundary between the L. lateralis fireflies from Korean and Japan. Phylogenetic analysis of the luciferase gene based on nucleotide sequences also separated the Korean from the Japanese $L$. lateralis (Fig. 5). As Japan and the Korea are separated by the Namhe Sea it is likely the genetic isolation is a consequence of geographic isolation.

In fact, other molecular studies on L. lateralis mt DNA also show genetic differences between Korean and Japanese samples. For example, Kim et al. (2000) found 2.9\% divergence in the mt $16 \mathrm{~S}$ rRNA sequences between two geographic samples, and emphasized that the degree of divergence is larger than that between two Luciola species, L. cruciata and L. owasai (1.5\%), endemic to Japan. Suzuki et al. (2004) also performed an RFLP analysis/direct sequencing of the $\mathrm{mt}$ COII gene of 46 Japanese and two Korean populations to test if the two Japanese ecological types, based on the flashing pattern, are associated with genetic differences. They found no evidence for them but did found an obvious genetic separation between the Japanese and Korean populations. Thus, the result of our study on the luciferase gene is consistent with the previous finding based on mt DNA. However, our results do not support the finding of Kim et al. (2001a) that the Muju and Boun populations of L. lateralis are genetically distinguishable on the basis of $\mathrm{mt}$ cytochrome oxidase I gene sequences. We found two distinguishable groups in the Muju samples and that the Boun samples have a closer relationship with one of the two Muju isoforms than they have to one another. To resolve this issue another molecular marker is required. Further, a more population-oriented study is expected in the future to confirm the geographic isolation between the L. lateralis populations in Korea and Japan.

In conclusion, we sequenced and found a novel isoform luciferase gene in the L. lateralis fireflies collected in Boun, Korea and compared its structure with that of other luciferase genes. This gene is identical in length and exon/intron structure in all L. lateralis. Phylogenetic analysis of the luciferase isoforms of the $L$. lateralis firefly appears to suggest that $L$. lateralis fireflies in Korea and Japan are genetically isolated.

ACKNOWLEDGEMENTS. This paper was supported by the Dong-A University Research Fund in 2003.

\section{REFERENCES}

Bailey J., Benard M. \& Burland T.G. 1994: A luciferase expression system for Physarum that facilitates analysis of regulatory elements. Curr. Genet. 26: 126-131.

Chо K.H., Lee J.S., Chо Y.D. \& Boo K.S. 1999: Structural polymorphism of the luciferase gene in the firefly, Luciola lateralis. Insect Mol. Biol. 8: 193-200.

Choi Y.S., Lee K.S., Bae J.S., Lee K.M., Kim S.R., Kim I., Lee S.M., SoHn H.D. \&. JIN B.R. 2002: Molecular cloning and expression of a cDNA encoding the luciferase from the firefly, Hotaria unmunsana. Comp. Biochem. Physiol. B. 132: 661-670.

Choi Y.S., Bae J.S., Lee K.S., Kim S.R., Kim I., Kim J.G., Kim K.Y., Kim S.E., Suzuki H., Lee S.M., Sohn H.D. \& Jin B.R. 2003: Genomic structure of the luciferase gene and phylogenetic analysis in the Hotaria-group fireflies. Comp. Biochem. Physiol. B. 134: 199-214.

Devine J.H., Kutuzova G.D., Green V.A., Ugarova N.N. \& BALDWIN T.O. 1993: Luciferase from the east European firefly Luciola mingrelica: cloning and nucleotide sequence of the cDNA, overexpression in Escherichia coli and purification of the enzyme. Biochim. Biophys. Acta 1173: 121-132. 
De Wet J.R., Wood K.W., Helinski D.R. \& Deluca M. 1985: Cloning of firefly luciferase cDNA and the expression of active luciferase in Escherichia coli. Proc. Natl. Acad. Sci. USA 82: 7870-7873.

De Wet J.R., Wood K.W., DeLuca M., Helinski D.R. \& SubraMANI S. 1987: Firefly luciferase gene: structure and expression in mammalian cells. Mol. Cell Biol. 7: 725-737.

Dilella A.G., Hope D.A., Chen H., Trumbauer M., Schwartz R.J. \& Sмітн R.G. 1988: Utility of firefly luciferase as a reporter gene for promoter activity in transgenic mice. Nucleic Acids Res. 16: 4159.

FelsensteIn J. 1985: Confidence limits on phylogenics: an approach using the bootstrap. Evolution 29: 783-791.

Felsenstein J. 1993: PHYLIP (Phylogeny Inference Package) ver. 3.5c. Department of Genetic, Univ. Washington, Seattle (on disk).

Howard P.K., Ahern K.G. \& Firtel R.A. 1988: Establishment of a transient expression system for Dictyostelium discoideum. Nucleic Acids Res. 16: 2613-2623.

Jacobs W.R., Barletta R.G., Udami R., Chan J., Kalkut G., Sosne G., Kieser T., Sarkis G.J., Hatfull G.F. \& Bloom B.R. 1993: Rapid assessment of drug susceptibilities of Mycobacterium tuberculosis by means of luciferase reporter phages. Science 260: 819-822.

Kim I., Lee S.C., Bae J.S., Jin B.R., Kim S.E., Kim J.G., Yoon H.J., Yang S.R., Lim S.H. \& SoHn H.D. 2000: Genetic divergence and phylogenetic relationships among the Korean fireflies, Hotaria papariensis, Luciola lateralis, and Pyrocoelia rufa (Coleoptera: Lampyridae), using mitochondrial DNA sequences. Korean J. Appl. Entomol. 39: 211-226.

Kim J.G., Kim I., Bae J.S., Jin B.R., Кiм K.Y., Кiм S.E., Сно J.Y., Chol Y.C., Lee K.Y., SoHN H.D. \& NoH S.K. 2001a: Genetic subdivision of the firefly, Luciola lateralis (Coleoptera: Lampyridae), in Korea determined by mitochondrial COI gene sequences. Korean J. Genetics 23: 203-219.

KIм J.G., Кім S.E., Сноі J.Y., Yoоn H.Y., Сног Y.C., Nobuyoshi O., Jin B.R. \& NoH S.K. 2001b: Developmental characteristics and life history of the Korean native firefly, Luciola lateralis. Int. J. Indust. Entomol. 3: 141-147.

Kondo T., Takahashi N. \& Muramatsu M. 1992: The regulation of the murine Hox-2.5 gene expression during cell differentiation. Nucleic Acids Res. 20: 5729-5735.

Lee K.S., Park H.J., Bae J.S., Goo T.W., Kim I., Sohn H.D. \& JIN B.R. 2001: Molecular cloning and expression of a cDNA encoding the luciferase from the firefly, Pyrocoelia rufa. $J$. Biotechnol. 92: 9-19.
Masuda T., Tatsumi H. \& Nakano E. 1989: Cloning and sequence analysis of cDNA for luciferase of a Japanese firefly, Luciola cruciata. Gene 77: 265-270.

McDermott F.A. 1964: The taxonomy of the Lampyridae (Coleoptera). Trans. Am. Entomol. Soc. 90: 1-72.

Miller A.J., Short S.R., Chua N.H. \& Say S.A. 1992: A novel circadian phenotype based on firefly luciferase expression in transgenic plants. Plant Cell 4: 1075-1087.

OhmiYa Y., Оhва N., Тон H. \& Tsuji F.I. 1995: Cloning, expression and sequence analysis of cDNA for the luciferases from the Japanese fireflies, Pyrocoelia miyako and Hotaria parvula. Photochem. Photobiol. 62: 309-313.

SuZUKI H. 1997: Molecular phylogenetic studies of Japanese fireflies and their mating systems (Coleoptera: Cantharoidea). Tokyo Metropol. Univ. Bull. Nat. Hist. 3: 1-53.

SuzuKi H. 2001: Studies on biological diversity of firefly in Japan. Int. J. Indust. Entomol. 2: 91-105.

SuzuKi H., Sato Y., Ohba N., Bae J.S., Jin B.R., Sohn H.D. \& KIM S.E. 2004: Phylogenetic analysis of the firefly, Luciola lateralis, in Japan and Korea based on mitochondrial cytochrome oxidase II gene sequences (Coleoptera: Lampyridae). Biochem. Genetics 42: (in press).

Swofford D.L. 1993: PAUP: Phylogenetic Analysis Using Parsimony, Ver. 3.1. Illinois Natural History Survey, Champaign (on disk).

Tatsumi H., Kajiyama K. \& Nakano E. 1992: Molecular cloning and expression in Escherichia coli of a cDNA clone encoding luciferase of a firefly, Luciola lateralis. Biochim. Biophys. Acta 1131: 161-165.

Vikas B.P., Sumathy S. \& Karumathil P.G. 1995: Baculovirus mediated high-level expression of luciferase in silkworm cells and larvae. BioTechniques 19: 97-104.

Wood K.V., Lam Y.A., Seliger H.H. \& McElroy W.D. 1989: Complementary DNA coding click beetle luciferase can elicit bioluminescence of different colors. Science 244: 700-702.

Ye L., Buck L.M., Schaeffer H.J. \& Leach F.R. 1997: Cloning and sequencing of a cDNA for firefly luciferase from Photuris pennsylvanica. Biochim. Biophys. Acta 1339: 39-52.

Zenno S., Shiraishi S., Inouye S. \& Saigo K. 1994: Cloning, Nucleotide Sequence and Expression of two cDNA Encoding for Luciferase from Photuris firefly. GenBank accession number U31240.

Received May 19, 2003; revised October 20, 2003; accepted December 7, 2003 